ARTÍCULO ORIGINAL

\title{
Un caso de resistencia de Rhipicephalus microplus (Acari: Ixodidae) al fipronil detectado en pruebas de campo en el este de Santiago del Estero, Argentina.
}

\author{
Nava $\mathrm{S}^{1,2^{*}}$, Morel $\mathrm{N}^{1}$, Mangold $A \mathrm{~J}^{1,2}$, Guglielmone $A \mathrm{~A}^{1}$ \\ ${ }^{1}$ Instituto Nacional de Tecnología Agropecuaria, Estación Experimental Agropecuaria Rafaela \\ (INTA - EEA Rafaela) \\ ${ }^{2}$ Consejo Nacional de Investigaciones Científicas y Técnicas (CONICET)
}

* Correspondencia: Santiago Nava. INTA - EEA Rafaela, CC 22, 2300 Rafaela, Santa Fe, Argentina

E-mail: nava.santiago@inta.gob.ar

Recibido: 30 Noviembre 2017. Aceptado: 15 Febrero 2018. Disponible en línea: 2 Marzo 2018

Editor: P. Beldomenico

RESUMEN. En este trabajo se describe la resistencia de una población Rhipicephalus microplus resistente al fipronil 1\% (ECTOLINE ${ }^{\circledR}$ ) en condiciones de campo en el este de Santiago del Estero, Argentina. Las pruebas fueron realizadas a modo de réplicas en dos establecimientos: "Establecimiento A" $y$ “Establecimiento B". En el Establecimiento A las diferencias en los niveles de infestación con garrapatas entre los grupos tratado y control nunca fueron estadísticamente significativas. Los porcentajes de eficacia oscilaron entre $27,3 \%$ y $69,3 \%$. En el Establecimiento B las diferencias entre los grupos tratado y control fueron significativas, con porcentajes de eficacia aumentando de $87,6 \%$ el día 2 post-tratamiento a 95,4\% el día 7 post-tratamiento. Las tasas de eclosión de los huevos originados de las teleóginas colectadas de los grupos tratados y control en el Establecimiento A fueron similares. La aplicación de fipronil 1\% en el Establecimiento B tuvo una eficacia global prácticamente absoluta, porque si bien la eficacia terapéutica no alcanzó el $100 \%$, la viabilidad reproductiva de las teleóginas obtenidas de los bovinos tratados fue nula. Los resultados del presente trabajo constituyen el primer registro publicado de una población de $R$. microplus resistente al fipronil $1 \%$ en condiciones de campo en Argentina.

SUMMARY. A case of resistance of Rhipicephalus microplus (Acari: Ixodidae) to fipronil detected in field trials in the east of Santiago del Estero, Argentina. The resistance of a population of Rhipicephalus microplus (Acari: Ixodidae) to fipronil 1\% (ECTOLINE ${ }^{\circledR}$ ) in the east of Santiago del Estero, Argentina, is described in this work. The trials were performed as replicates in two establishments: "Establishment A" and "Establishment B". The differences in the level of tick infestation between treated and control groups in the Establishment A were not significant. The efficacy percentage ranged from $27.3 \%$ to $69.3 \%$. Contrarily, the differences between treated and control groups in the Establishment B were significant, with efficacy percentage increasing from $87.6 \%$ (day 2 posttreatment) to $95.4 \%$ (day 7 post-treatment). The eclosion rates of the eggs produced by the engorged females collected in both treated and control groups in Establishment A were similar. The application of fipronil $1 \%$ in the Establishment B had an efficacy almost absolute, because even though the therapeutic efficacy did not reach $100 \%$, the reproductive viability of the engorged females collected on the treated cattle was null. The results of this work constitute the first published record of a population of $R$. microplus resistant to fipronil $1 \%$ under field conditions in Argentina.

Palabras clave: resistencia, garrapatas, fipronil, Argentina

Key words: resistance, ticks, fipronil, Argentina

\section{Introducción}

Rhipicephalus microplus es la especie de garrapata con mayor relevancia mundial para la producción pecuaria (Jongejan y Uilemberg, 2004). En Argentina, en las zonas ubicadas al norte de los paralelos $30 \circ-310 \mathrm{~S}, R$. microplus ocasiona fuertes limitaciones al desarrollo de la ganadería de carne y leche (Guglielmone y Nava, 2013). 
El método de control para $R$. microplus más empleado se basa en la aplicación de garrapaticidas químicos. El uso intensivo de estos acaricidas ha resultado en muchos casos en la aparición de poblaciones de garrapatas resistentes al producto utilizado. En el continente sudamericano ya se han detectado casos de resistencia a prácticamente todos los compuestos químicos garrapaticidas disponibles comercialmente (Guglielmone et al., 2006; Castro-Janer et al., 2010a,b; Guerrero et al., 2012; Lovis et al., 2013; Reck et al., 2014). Particularmente en Argentina, hasta el momento se han registrado poblaciones de $R$. microplus resistentes a las drogas arsenicales, organofosforados, piretroides y formamidinas en las provincias argentinas de Corrientes, Córdoba, Chaco, Santiago del Estero, Salta y Santa Fe (Grillo-Torrado y Pérez-Arrieta, 1977; Mangold et al., 2000, 2004; Guglielmone et al., 2006; Cutullé et al., 2013).

Uno de los grupos químicos aprobados como garrapaticidas en Argentina por el Servicio Nacional de Sanidad y Calidad Agroalimentaria (SENASA) son los fenilpirazoles, representados por el fipronil $1 \%$ (SENASA- http://www.senasa.gob.ar/garrapaticidasautorizados-para-uso-en-zonas-de-control-zona-sucia). El mismo está indicado para ser utilizado en la "Zona de Control" (ver detalles de la zonificación en la resolución 382-E/2017 del SENASA -

http://www.senasa.gob.ar/normativas/resolucion-3822017-senasa-servicio-nacional-de-sanidad-y-calidadagroalimentaria). Casos de resistencia de $R$. microplus al fipronil en Sudamérica fueron registrados en Brasil y Uruguay (Cuore et al., 2007; Castro-Janer et al., 2010 a,b; Klafke et al., 2016). Recientemente, tras la realización de pruebas de campo para controlar la infestación con $R$. microplus en bovinos en el noroeste de Argentina, se observó una eficacia inferior a la esperada en una población de esta garrapata luego del tratamiento con fipronil 1\% en el este de Santiago del Estero, Argentina. A continuación se presentan y analizan los resultados de la evaluación de fipronil $1 \%$ en dicha población de $R$. microplus.

\section{Materiales y Métodos}

Dos pruebas para mensurar la eficacia del fipronil $1 \%$ para el control de $R$. microplus en el este de Santiago del Estero fueron realizadas a modo de réplicas en dos establecimientos (nombrados en este trabajo como "Establecimiento A" y "Establecimiento B") ubicados en la localidad de Quimilí, departamento Moreno, Santiago del Estero. El sitio de estudio pertenece a la eco-región del Chaco Seco (Burkart et al., 2009), donde la vegetación xerófila original ha sufrido una transformación significativa, debido a la deforestación de los bosques nativos para la introducción de pasturas megatérmicas, principalmente de Panicum maximum var. Gatton panic. El Establecimiento A representa una unidad dedicada a la ganadería de invernada con ingreso constante de bovinos de distintos orígenes geográficos, mientras que la ganadería de cría bovina es la principal actividad pecuaria del Establecimiento B. El historial de empleo de fipronil como garrapaticida en ambos establecimientos comprende los últimos 5 años, el cual es aplicado con una frecuencia de dos a tres tratamientos anuales.

Dos grupos de 10 bovinos de raza Braford (Establecimiento A: novillitos de 15 meses de edad; Establecimiento B: vaquillonas de 27 meses de edad), con niveles similares de infestación con $R$. microplus ( $p>0.5$ ) fueron evaluados en cada una de las pruebas. Uno de los grupos fue tratado con una formulación comercial de fipronil $1 \%$ de aplicación por derrame dorsal (ECTOLINE ${ }^{\circledR}$, Merial Argentina S.A.) con la dosis indicada por el fabricante de $1 \mathrm{ml} / 10 \mathrm{~kg}$. El segundo grupo de animales no fue tratado a fin de ser adoptado como grupo control. Los bovinos incluidos en los grupos tratados fueron pesados para ajustar de manera exacta la dosificación del garrapaticida en cada individuo. En ambos establecimientos se utilizó la misma partida de ECTOLINE ${ }^{\circledR}$ para evitar un sesgo en los resultados debido a factores inherentes a la fabricación del garrapaticida.

Durante las pruebas los grupos se mantuvieron separados en potreros similares (tamaño, disponibilidad de agua, tipo y estados de las pasturas) distanciados entre sí por al menos 5 metros. Se contabilizaron para cada bovino las hembras de $R$. microplus de 4,5-8,0 mm de largo presentes en la mitad izquierda, y el número obtenido fue duplicado para el análisis estadístico. La diferencia en los niveles de infestación con $R$. microplus entre los grupos se evaluó durante los 7 días posteriores al tratamiento utilizando la prueba no paramétrica de Kruskal-Wallis, y la eficacia terapéutica con respecto a los controles se determinó con la formula modificada de Abbot descripta en Henderson y Tilton (1955). Para determinar los efectos limitantes del tratamiento con fipronil $1 \%$ sobre parámetros bioló-gicos de las garrapatas, se colectaron teleóginas de los cuatro grupos de bovinos, se pesaron y luego se mantuvieron en el laboratorio a 25 ㅇ C, $80-83 \%$ de humedad relativa, y en un fotoperíodo de 12 horas luz - 12 horas oscuridad. Se registraron el peso de las teleóginas y la tasa de eclosión de los huevos, y se calcularon el índice de eficiencia reproductiva (IER: número de huevos/ miligramo de peso de la teleógina) (Drummond y Whetstone, 1970) y el índice de fertilidad (IF: número de larvas eclosionadas/ miligramo de peso de la teleógina) (Aguirre et al., 2005). Las diferencias en los valores de estos parámetros biológicos entre los dos grupos tratados y los dos controles se compararon mediante el análisis de la varianza (ANOVA), empleándose para las comparaciones a posteriori la prueba de Tukey (Zar, 1999). 


\section{Resultados}

Los resultados referidos a la eficacia terapéutica obtenida con los tratamientos de los bovinos con fipronil $1 \%$ en las dos pruebas se muestran en la tabla 1. En el Establecimiento A, las diferencias en los niveles de infestación con garrapatas entre los grupos tratado y control nunca fueron estadísticamente significativas (Tabla 1). Asimismo, los porcentajes de eficacia no superaron el umbral del $70 \%$. Los valores oscilaron entre $27,3 \%$ y $69,3 \%$ (Tabla1). Contrariamente, en el Establecimiento $\mathrm{B}$ las diferencias entre los grupos tratado y control fueron significativas a partir del día 2 post-tratamiento, con porcentajes de eficacia aumentando de $87.6 \%$ el día 2 post-tratamiento a $95.4 \%$ el día 7 post-tratamiento (Tabla 1 ).

Tabla 1: Niveles de infestación con Rhipicephalus microplus en los grupos de bovinos tratados con fipronil $1 \%$ (GT) y en los grupos control (GC) en los establecimientos A y B de la localidad de Quimilí, Santiago del Estero, Argentina. Se indican los valores de la media (mínimos y máximos entre paréntesis) y los porcentajes de eficacia del tratamiento. DPT: día post tratamiento.

\begin{tabular}{|c|c|c|c|c|}
\hline$\frac{\text { Establecimiento }}{\underline{\mathrm{A}}}$ & $\underline{\mathbf{G T}}$ & $\underline{\mathrm{GC}}$ & & \\
\hline DPT & Media* & Media* & $p^{* *}$ & \%Eficacia \\
\hline 0 & $\begin{array}{c}46.2 \\
(20-80)\end{array}$ & $\begin{array}{c}45.6 \\
(24-78)\end{array}$ & 0.940 & - \\
\hline 1 & $\begin{array}{c}25.4 \\
(10-48)\end{array}$ & $\begin{array}{c}38.2 \\
(8-88)\end{array}$ & 0.448 & 34.7 \\
\hline 2 & $\begin{array}{c}19.6 \\
(4-46)\end{array}$ & $\begin{array}{c}30.8 \\
(8-60)\end{array}$ & 0.212 & 37.2 \\
\hline 3 & $\begin{array}{c}30.8 \\
(4-68)\end{array}$ & $\begin{array}{c}41.8 \\
(10-82)\end{array}$ & 0.405 & 27.3 \\
\hline 7 & $\begin{array}{c}3.8 \\
(0-18)\end{array}$ & $\begin{array}{c}12.2 \\
(0-50)\end{array}$ & 0.165 & 69.3 \\
\hline$\frac{\text { Establecimiento }}{\text { B }}$ & GT & $\underline{\mathrm{GC}}$ & & \\
\hline DPT & Media* & Media* & $p^{* *}$ & \%Eficacia \\
\hline 0 & $\begin{array}{c}81.0 \\
(26-168)\end{array}$ & $\begin{array}{c}78.5 \\
(40-146)\end{array}$ & 0.789 & - \\
\hline 1 & $\begin{array}{c}50.5 \\
(6-132)\end{array}$ & $\begin{array}{c}90.2 \\
(36-138)\end{array}$ & 0.06 & 50.2 \\
\hline 2 & $\begin{array}{c}11.7 \\
(4-36)\end{array}$ & $\begin{array}{c}91.7 \\
(56-124)\end{array}$ & $<0.01$ & 87.6 \\
\hline 3 & $\begin{array}{c}4.7 \\
(2-8)\end{array}$ & $\begin{array}{c}64.7 \\
(38-90)\end{array}$ & $<0.01$ & 93 \\
\hline 7 & $\begin{array}{c}1 \\
(0-8)\end{array}$ & $\begin{array}{c}21.3 \\
(8-40)\end{array}$ & $<0.01$ & 95.4 \\
\hline $\begin{array}{l}\text { * Media: número d } \\
\text { nados } \\
\text { ** Prueba no parar } \\
\text { tamiento. }\end{array}$ & garrapatas & húmero d & $\begin{array}{l}\text { boving } \\
\text { DPT: }\end{array}$ & $\begin{array}{l}\text { exami- } \\
\text { post-tra- }\end{array}$ \\
\hline
\end{tabular}

Se colectaron teleóginas de los dos grupos controles y del grupo tratado en el Establecimiento $A$ en todas cuentas realizadas entre los días 1 y 7 post-tratamiento, mientras que en el grupo tratado del Establecimiento $B$ sólo se colectaron teleóginas los días 1 y 2 post-tra- tamiento. El tratamiento con fipronil 1\% disminuyó significativamente el peso de las teleóginas en los dos establecimientos, pero la tasa de eclosión sólo se vio afectada en los huevos obtenidos de las teleóginas colectadas sobre los bovinos tratados en el Establecimiento $\mathrm{B}$, donde el valor de la misma fue cero (Tabla 2). Aunque los valores de IER e IF fueron significativamente menores en el grupo tratado del Establecimiento $\mathrm{A}$ en comparación con aquellos observados en los dos grupos controles, la tasa de eclosión de los huevos originados de las teleóginas colectadas de los bovinos tratados en el Establecimiento A fue similar a la observada en los huevos obtenidos de las teleóginas colectadas de los grupos controles en ambos establecimientos (Tabla 2).

Tabla 2: Valores del peso de las teleóginas de Rhipicephalus microplus, de la tasa de eclosión de los huevos y de los índices de eficiencia reproductiva (IER) y de fertilidad (IF) (ver definición de los índices en la sección materiales y métodos). EAGT: grupo tratado con fipronil $1 \%$ en Establecimiento A; EAGC: grupo control en Establecimiento A; EBGT: Grupo tratado con fipronil $1 \%$ en Establecimiento B; EBGC: grupo control en Establecimiento B. Se presentan los valores de la media y entre paréntesis el desvío estándar. Los números que no comparten superíndices son significativamente diferentes $(P<$ 0,01 ) en el ANOVA

\begin{tabular}{|ccccc|}
\hline \multirow{2}{*}{ Peso (mg) } & EAGT & EAGC & EBGT & EBGC \\
& $137,4 \mathrm{a}$ & $196,5 \mathrm{~b}$ & $136,7 \mathrm{~b}$ & $187,2 \mathrm{a}$ \\
& $( \pm 13,8)$ & $( \pm 15,1)$ & $(14,4)$ & $( \pm 15,3)$ \\
\hline \% eclosión & $97,8 \mathrm{a}$ & $98,9 \mathrm{a}$ & 0 & $97,4 \mathrm{a}$ \\
& $( \pm 0,6)$ & $( \pm 0,4)$ & & $( \pm 0,7)$ \\
\hline IER & $4,7 \mathrm{a}$ & $7,8 \mathrm{~b}$ & - & $7,6 \mathrm{~b}$ \\
& $( \pm 0,4)$ & $( \pm 0,6)$ & & $( \pm 0,4)$ \\
\hline IF & $4,5 \mathrm{a}$ & $7,6 \mathrm{~b}$ & - & $7,5 \mathrm{~b}$ \\
& $( \pm 0,4)$ & $( \pm 0,6)$ & & $( \pm 0,4)$ \\
\hline
\end{tabular}

Se puede afirmar que la aplicación de fipronil $1 \%$ en el Establecimiento $\mathrm{B}$ tuvo una eficacia global prácticamente absoluta, porque si bien la eficacia terapéutica no alcanzó el $100 \%$, la viabilidad reproductiva de las teleóginas obtenidas de los bovinos tratados fue nula.

\section{Discusión}

La resistencia de las garrapatas a los acaricidas es un fenómeno hereditario que se define como una reducción en la susceptibilidad de las mismas a un compuesto químico cuando éste es aplicado en la concentración recomendada y respetando las recomendaciones para su uso (FAO, 2004). El análisis realizado en el presente estudio de los valores de porcentaje de eficacia, de la tasa de eclosión de los huevos y de los IER e IF, sugiere fuertemente que la población de $R$. microplus sujeta a tratamiento en el Establecimiento $A$ es resistente al fipronil $1 \%$. Aunque se observó una reducción en los niveles de infestación del grupo tratado en relación al grupo control en el Establecimiento A, la misma no fue significativa y los porcentajes de eficacia fueron bajos 
(Tabla 1). La presencia de teleóginas en todas las cuentas llevadas a cabo durante los 7 días posteriores al tratamiento y la viabilidad de sus huevos, la cual se ve expresada en una tasa de eclosión cercana al 98\%, brindan un sustento adicional a la hipótesis de la existencia de una población de $R$. microplus resistente al fipronil en el este de Santiago del Estero. Sería conveniente complementar estos resultados con pruebas in vitro.

A diferencia de lo observado en el Establecimiento A, el tratamiento de los bovinos con la misma partida de fipronil $1 \%$ en el Establecimiento B permitió alcanzar altos niveles de eficacia terapéutica. Los porcentajes de eficacia mostraron una tendencia creciente entre el día 1 y 7 post-tratamiento, donde se alcanzó un valor máximo de $95.4 \%$ (Tabla 1). Sumado a esto, las teleóginas colectadas del grupo tratado los días 1 y 2post-tratamiento (no se colectaron los días 3 y 7 posttratamiento) no fueron viables debido a que la tasa de eclosión de sus huevos fue cero.

El historial de uso de fipronil 1\% para controlar garrapatas en los Establecimientos A y B es similar, hecho que dificulta en una primera instancia atribuir la aparición de resistencia a la frecuencia de tratamientos. La generación de resistencia en un establecimiento dado también puede deberse al ingreso de ganado con garrapatas provenientes de otros predios donde la frecuencia de genotipos resistentes en las poblaciones de este parásito es mayor. En este sentido, el manejo del Establecimiento A (ganadería de invernada con ingreso constante de bovinos de distintos orígenes geográficos) lo hace más vulnerable a la introducción de garrapatas resistentes que el Establecimiento B, cuya actividad ganadera de cría implica un menor ingreso de bovinos de otros predios. El desarrollo de futuras investigaciones centradas en indagar sobre los procesos que determinan la generación y diseminación de la resistencia a los garrapaticidas químicos en Argentina contribuirían a testear las hipótesis planteadas en este párrafo.

Los resultados del presente trabajo constituyen el primer registro publicado de una población de $R$. microplus resistente al fipronil $1 \%$ en Argentina, el cual se suma a los hallazgos previos de garrapatas resistentes a este compuesto químico en Brasil y Uruguay (Cuore et al., 2007; Castro-Janer et al., 2010 a,b; Klafke et al., 2016). La emergencia de poblaciones de garrapatas resistentes no solo al fipronil sino también a otros grupos químicos en Argentina, Brasil y Uruguay, donde la ganadería ocupa una lugar de relevancia en la actividad económica, resalta la necesidad de desarrollar métodos de control de $R$. microplus que alcancen una eficacia productivamente sustentable minimizando la frecuencia de tratamientos con acaricidas químicos. Esto constituye un desafío considerable y probablemente se requiera el desarrollo de técnicas alternativas que no dependan exclusivamente del tratamiento con garrapaticidas químicos.

\section{Agradecimientos}

Este trabajo se realizó con el apoyo financiero de INTA, Asociación Cooperadora INTA Rafaela y Agencia Nacional de Promoción Científica y Tecnológica (PICT 0676 y PICT 0550).

\section{Bibliografía}

Aguirre DH, Gaido AB, Cafrune MM, Castelli ME, Mangold AJ, Guglielmone AA. 2005. Eprinomectin pour-on for control of Boophilus microplus (Canestrini) ticks (Acari: Ixodidae) on cattle. Vet. Parasitol. 127: 157-163.

Burkart R, Bárbaro O, Sánchez RO, Gómez DA. 1999. Ecoregiones de la Argentina. Administración de Parques Nacionales, Secretaría de Recursos Naturales y Desarrollo Sustentables, Buenos Aires, 42 pp.

Castro-Janer E, Martins JR, Mendes MC, Namindome A, Klafke GM, Schumaker TTS. 2010a. Diagnoses of fipronil resistant in Brazilian cattle ticks (Rhipicephalus (Boophilus) microplus) using in vitro larval bioassays. Vet. Parasitol. 173: 300-306.

Castro-Janer E, Rifran L, González P, Piaggio J, Gil A, Schumaker TTS. 2010b. Rhipicephalus (Boophilus) microplus (Acari: Ixodidae) resistance to fipronil in Uruguay evaluated by in vitro bioassays. Vet. Parasitol. 169: 172-177.

Cuore U, Trelles A, Sanchis J, Gayo V, Solari MA. 2007. Primer diagnóstico de resistencia al fipronil en la garrapata común del ganado Boophilus microplus. Veterinaria (Montevideo) 42: 165-166.

Cutullé C, Lovis L, D’Agostino BI, Balbiani GG, Morici G, Citroni D, Reggi J. Caracostantogolo JL, 2013. In vitro diagnosis of the first case of amitraz resistance in Rhipicephalus microplus in Santo Tomé (Corrientes), Argentina. Vet. Parasitol. 192: 296300.

Drummond RO, Whetstone TM. 1970. Oviposition of the gulf coast tick. J. Econ. Entomol. 63: 1547-1551.

FAO, 2004. Resistance Management and Integrated Parasite Control in Ruminants - Guidelines, Module 1 - Ticks: Acaricide Resistance: Diagnosis, Management and Prevention. Food and Agriculture Organization, Animal Production and Health Division, Rome, pp. 25-77.

Grillo Torrado JM, Pérez Arrieta A. 1977. Nuevo tipo de fósforo-resistencia en la garrapata común del ganado bovino (Boophilus microplus) en la República Argentina. Rev. Med. Vet. 58: 101-105

Guerrero FD, Lovis L, Martins JR. 2012. Acaricide resistance mechanisms in Rhipicephalus (Boophilus) microplus. Rev. Bras. Parasitol. Vet: 21, 1-6.

Guglielmone AA, Nava S. 2013. Epidemiología y control de las garrapatas de los bovinos en la Argentina. En: Enfermedades Parasitarias con Importancia Clínica y Productiva en Rumiantes: Fundamentos Epidemiológicos para su Diagnóstico 
y Control (ed. A. Nari y C.Fiel), Editorial Hemisferio Sur, Buenos Aires. Pp. 441-456.

Guglielmone AA, Mangold AJ, Castelli M, Suárez VH, Aguirre $\mathrm{DH}$, Alcaraz E, Cafrune MM, Cetrá B, Fader OW, Luciani CA, Medus PD, Nava S. 2006. Toxicidad in vitro de la cipermetrina para Rhipicephalus (Boophilus) microplus (Can.) y del diazinón para Haematobia irritans (L.) en la Argentina. Rev. Inv. Agrop. 35: 31-41.

Henderson CF, Tilton EW. 1955. Tests with acaricides against the brow wheat mite. J. Econ. Entomol. 48:157-161.

Jongejan F, Uilenberg G. 2004. The global importance of ticks. Parasitology: 129, 1-12.

Klafke GM, Webster A, Dall'Agnol B, Pradel E, Silva J, de La Canal LH, Becker $M$, Osório MF, Mansson $M$, Barreto $R$, Scheffer R, Souza UA, Corassini VB, dos Santos J, Reck J, Martins JR, 2016. Multiple resistance to acaricides in field populations of Rhipicephalus microplus from Rio Grande do Sul state, southern Brazil. Ticks Tick-borne Dis. 8: 73-80.

Lovis L, Mendes MC, Perret JL, Martins JR, Bouvier J, Betschart B, Sager H. 2013. Use of the Larval Tarsal Test to determine acaricide resistance in Rhipicephalus (Boophilus) microplus Brazilian field populations. Vet. Parasitol. 191: 323-331.

Mangold A J, Muñoz Cobeñas ME, Castelli MC, Scherling NJ, Delfino M, Guglielmone AA. 2000. Resistencia a la cipermetrina en una población de Boophilus microplus (Acari: Ixodidae) del norte de Santa Fe, Argentina. Rev. Med. Vet. (Bs. As.), 81: 259261.

Mangold AJ, Castelli ME, Nava S, Aguirre DH, Guglielmone AA. 2004. Poblaciones de la garrapata Rhipicephalus (Boophilus) microplus resistentes a los piretroides en Córdoba y Salta, Argentina. Revista FAVE - sección Ciencias Veterinarias 3: 5559.

Reck J, Klafke GM, Webster A Dall'Agnol B, Scheffer R, Souza UA, Corassini VB, Vargas R, Santos JS, Martins JR. 2014. First report of fluazuron resistance in Rhipicephalus microplus: field tick population resistant to six classes of acaricides. Vet. Parasitol. 201: 128-136

Spath EJA, Guglielmone AA, Signorini AR, Mangold AJ. 1994. Estimación de las pérdidas económicas directas producidas por la garrapta Boophilus microplus y las enfermedades asociadas en la Argentina. 1ra parte. Therios 23: 341-360.

Zar JH. 1999. Biostatistical analysis. 4ta ed., Prentice-Hall, New Jersey, USA. 663 pp. 